\title{
MAGNETIC PROPERTIES OF EuS/PbS SEMICONDUCTING STRUCTURES
}

\author{
A. Stachow-Wójcik, A. Twardowski \\ Institute of Experimental Physics, Warsaw University \\ Hoża 69, 00-681 Warsaw, Poland \\ T. Story, W. Dobrowolsiki, E. Grodzicka \\ Institute of Physics, Polish Academy of Sciences \\ Al. Lotników 32/46, 02-668 Warsaw, Poland
}

and A. Sipatow

Kharkov State Technical University, Kharkov, Ukraine

\begin{abstract}
We report results of magnetization study of $\mathrm{EuS} / \mathrm{PbS}$ superstructures with different thicknesses of magnetic and nonmagnetic layers. Reduction of ferromagnetic phase transition temperature was found with decreasing EuS thickness. Reasonable description of this effect is obtained within the model based on the mean field approximation.
\end{abstract}

PACS numbers: 75.50.Pp

The superstructures built of magnetic epilayers intercalated with nonmagnetic material have recently attracted considerable interest. They offer attractive possibilities to study dimensional effects (3D to $2 \mathrm{D}$ crossover) as well as coupling between magnetic layers via nonmagnetic medium. The structures of EuS/PbS can be regarded as a model Heisenberg-type ferromagnet/semiconductor superstructure. The collective magnetic behavior of bulk EuS is governed by ferromagnetic (F) nearest neighbors (NN) interaction $\left(J_{1}=+0.22\right)$ and antiferromagnetic (AF) next nearest neighbors coupling $\left(J_{2}=-0.11\right)$ [1]. In effect EuS orders ferromagnetically at $T_{\mathrm{c}}=16.6 \mathrm{~K}$ [1]. Electronically EuS is a semi-insulator [1] and $\mathrm{PbS}$ a degenerated semiconductor [2]. Moreover EuS and $\mathrm{PbS}$ both occur in the rock salt structure with nearly perfect lattices matching $(\Delta a / a=0.6 \%)$ [3]. These features make EuS/PbS especially attractive for studying their magnetic properties as a function of layer thickness and electronic properties of nonmagnetic $\mathrm{PbS}$.

The epitaxial layers of EuS/PbS were grown from vapor phase on monocrystalline $\mathrm{BaF}_{2}$ (111) or $\mathrm{KCl}(001)$ substrates, with $\mathrm{PbS}$ buffer layer. EuS was evaporated using the electron gun. The obtained structures were checked by X-ray 
diffraction in order to determine the superlattice period. We studied a set of structures with EuS thicknesses ranging from $6 \AA$ to $700 \AA$ and $\mathrm{PbS}$ from $20 \AA$ to $600 \AA$. Magnetization of the structures was measured using a SQUID magnetometer in the temperature range of $2-100 \mathrm{~K}$ and magnetic field up to $6 \mathrm{~T}$. The magnetic field was in the plane of the epilayers.

The temperature dependence of low field $(0.001 \mathrm{~T})$ magnetization clearly shows a paramagnetic-ferromagnetic phase transition. The representative result is displayed in Fig. 1a. Since magnetization obeys mean field (MFA) prediction $M \approx\left(T_{\mathrm{c}}-T\right)^{1 / 2}$, this formula was used to evaluate phase transition temperature (Fig. 1b). Alternatively transition temperature was determined from the magnetization $M(T)$ inflection point. Both methods produced very similar results. The determined ferromagnetic Curie temperature strongly depends on magnetic EuS and nonmagnetic $\mathrm{PbS}$ epilayers thickness. In Fig. 2 we show magnetic layer thickness dependence for EuS-PbS/ $\mathrm{BaF}_{2}$ structures, for which EuS layer thicknesses varied from 2 to 23 monolayers (MLs), while $\mathrm{PbS}$ layers were kept nearly constant $(150-175 \AA)$. It is clear that for EuS thicker than roughly $10 \mathrm{ML} T_{\mathrm{c}}$ is nearly the same, but appreciably lower than for bulk EuS: $13.6 \mathrm{~K}$ versus $16.6 \mathrm{~K}$. We ascribe this lowering of the critical temperature to the residual strain present in our structures. Whether it results from $\mathrm{EuS} / \mathrm{PbS}$ or superstructure/substrate mismatch is not entirely clear at the moment.

For the structures with EuS layers thinner than roughly $10 \mathrm{ML} T_{\mathrm{c}}$ decreases with decreasing EuS layer thickness (Fig. 2). We attribute this effect primarily to the reduction of the number of magnetic neighbors at the superstructures. Even for sharp EuS/PbS interface spins in the two outermost MLs have only 9 NN (less on average if the number of MLs is less than 2), instead of $12 \mathrm{NN}$ for bulk. For $\mathrm{EuS} / \mathrm{PbS}$ interfaces with finite widths, the intermixing of $\mathrm{EuS}$ with $\mathrm{PbS}$ reduces the average number of magnetic neighbors even further.

We considered this effect using the so-called "bond-loss model" [4], which assumes that the phase transition tempcrature for a superstructure $T_{c}^{\prime}$ scales by the average number of magnetic neighbors: $T_{\mathrm{c}}^{\prime}=T_{\mathrm{c}}^{\text {bulk }}\left(z_{1} J_{1}+z_{2} J_{2}\right) /\left(12 J_{1}+6 J_{2}\right)$, where $z_{1}$ and $z_{2}$ are the average numbers of NN (coupled by $J_{1}$ ) and NNN (coupled by $J_{2}$ ), respectively. In Fig. 2 we show prediction of this approach for a sharp interface, as well as for sample with a non-sharp interface. In the latter case $2 \mathrm{ML}$ interface with linear profile was assumed. For both sharp and non-sharp interfaces $T_{\mathbf{c}}^{\text {bulk }}=13.6 \mathrm{~K}$ was adopted, as resulting from thick EuS layers samples. As may be noticed a reasonable description of the data may be obtained for rather a sharp interface, extending for about $1 \mathrm{ML}$. This finding is in agreement with X-ray experiments; which suggest the sharp EuS/PbS interface, of the order of $1 \mathrm{ML}$.

We note that the above approach ignores the strain effects (except reduction of $T_{\mathrm{c}}^{\text {bulk }}$ ), which may be important. The substrate/superstructure mismatch influences strongly $T_{\mathrm{c}}^{\text {bulk }}\left(=13.6 \mathrm{~K}\right.$ for $\mathrm{BaF}_{2}$ substrate and $17.3 \mathrm{~K}$ for $\left.\mathrm{KCl}\right)$. Also EuS/PbS misfit, although relatively small, may influence $T_{\mathrm{c}}$ substantially. These effects will be discussed elsewhere.

Finally we note a strong effect of the nonmagnetic $\mathrm{PbS}$ layer thickness on the paramagnetic-ferromagnetic transition. Generally speaking the thinner $\mathrm{PbS}$ layer the lower critical temperature, as low as about $8 \mathrm{~K}$ for $40 \AA \mathrm{EuS} / 25 \AA \mathrm{PbS}$ on 

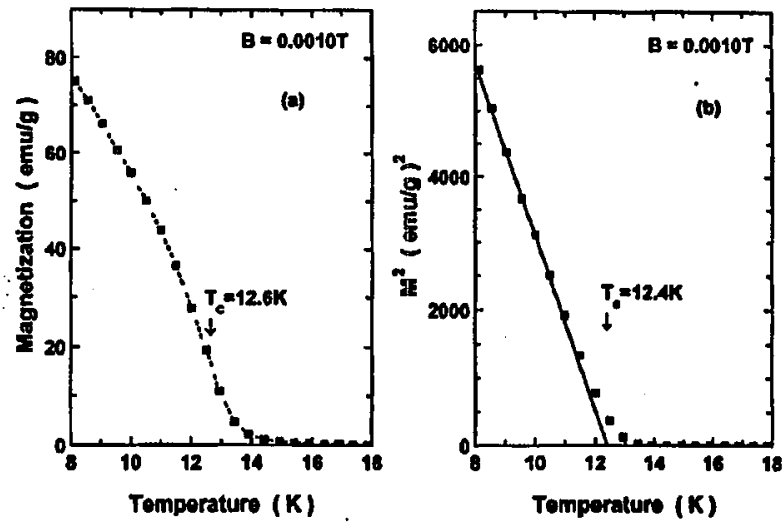

Fig. 1. (a) Magnetization at $B=0.001 \mathrm{~T}$ of $\operatorname{EuS}(55 \AA)-\operatorname{PbS}(175 \AA)$ grown on (111) $\mathrm{BaF}_{2}$ substrate as a function of temperature. The line is to guide the eye only. (b) The same data plotted as squared magnetization versus temperature. The straight line corresponds to the mean field prediction for a ferromagnet $M \approx\left(T_{\mathrm{c}}-T\right)^{1 / 2}$. The extrapolated critical temperature is in this case $T_{c}=12.4 \mathrm{~K}$.

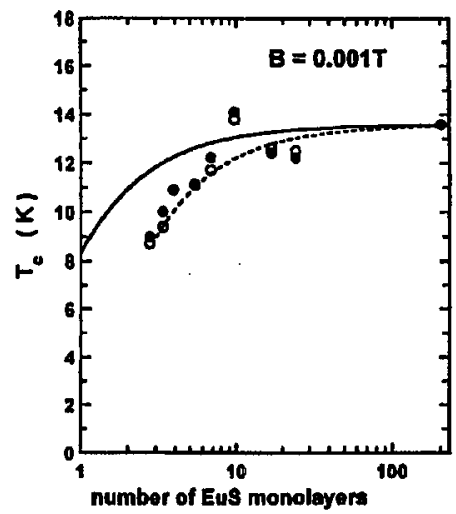

Fig. 2. Critical temperature $T_{\mathrm{c}}$ of EuS-PbS/BaF 2 as a function of EuS layer thickness, expressed in EuS monolayers. The thickness of $\mathrm{PbS}$ layers is nearly constant (44-52 ML). Full points denote $T_{\mathrm{c}}$ obtained from MFA extrapolation, while empty symbols show $T_{\mathrm{c}}$ resulting from the magnetization $M(T)$ function inflection point. The lines represent the results of the model described in the text. Solid line: sharp EuS-PbS interface, broken line: interface extending for 2 monolayers, with linear profile. $T_{c}^{\text {bulk }}=13.6 \mathrm{~K}$ was assumed.

$\mathrm{KCl}$. The observed behavior may reflect the magnetic interlayer coupling. Further studies are necessary to clarify the actual situation.

This work was supported by the Committee for Scientific Research, in particular under grant No. 2 P03B 10912. 


\section{References}

[1] A. Mauger, C. Godart, Phys. Rep. 41, 51 (1986).

[2] G. Nimtz, B. Schlicht, R. Dornhaus, Narrow Gap Semiconductors, Springer, Berlin 1983.

[3] I.V. Kolesnikov, A.Yu. Sipatov, Sov. Phys. Semicond. 26, 598 (1989).

[4] D. Kostyk, Y. Shapira, E.J. McNiff, Jr., T.Q. Vu, A. Twardowski, Solid State Commun. 92, 473 (1994); M.E. Fisher, in: Proc. of Interat. School of Physics "Enrico Fermi", Course LI, Critical Phenomena, Ed. M.S. Green, Academic, New York 1971 , p. 1. 